\title{
Mathematical Modeling for the Transmission of Ebola Virus
}

\author{
Anqi Li \\ Department of Electrical Engineering, North China Electric Power University, Baoding 071000, \\ China
}

sumer951112@163.com

Keywords: SEIR model; Ebola transmission model; Grey Prediction mode

\begin{abstract}
The goal of this model is to optimize the eradication of Ebola, which has been increasingly severe since 2014, causing more than 8966 deaths. Without effective control, human society will face a serious threat. This paper primarily addresses the problem by predicting the trend of Ebola transmission using the modified SIR model-SEIR model. Based on the data from the WHO, the parameters of this model are obtained. From this, epidemic situation in the future can be clearly observed. Besides, relatively accurate data in the short term can be gotten through this Grey Prediction model, which remedies the shortage of SEIR model in the short-term prediction. Thus, Ebola epidemic situation is bound to be eased and possibly be eradicated within a certain period.
\end{abstract}

\section{Introduction}

Ebola virus, a much more threatening virus than SARS, with a death rate of between $50 \%$ and $90 \%$, have intruded into this lives. Its outbreak in west Africa, especially in Guinea , Liberia and Sierra Leone, have recently draw the attention from all over the world. Now, the world medical association has announced that their new medication could stop Ebola and cure patients whose diseases are not advanced. Then, can this news bring luckiness to the people suffering from this horrible disease? Here, this paper will build a realistic and sensible model to solve the distribution of the drug, location of delivery considering the transmission of Ebola and speed of manufacturing the drug or the vaccine. Through this model, the tensity of fighting with Ebola at present can be relieved, which may eradicate it in short run. This paper manages to collect data to explore the regularity of Ebola transmission and predict the trend in the future.

\section{Ebola Transmission Model}

\section{1 Establishment of SEIR model}

To decide a optimal distribution system, this paper first need to determine the demanding quantity. That is, according to the analysis of data , this paper get the Ebola transmission regularity and then this paper predict the disease out-breaking condition in those countries in the future. To reach this, this paper first build the Ebola transmission model. The most universe and adequate model nowadays used in the fields of infectious diseases is the SIR model and it can be modified according to the actual disease condition. And here this paper take incubation period and death into consideration additionally. Thus, this paper build the differential model — SEIR model and set these equations 


$$
\left\{\begin{array}{c}
\frac{\mathrm{dS}_{(\mathrm{t})}}{\mathrm{dt}}=-\lambda \mathrm{I}_{(\mathrm{t})} \mathrm{S}_{(\mathrm{t})} \\
\frac{\mathrm{dE}_{(\mathrm{t})}}{\mathrm{dt}}=\lambda \mathrm{I}_{(\mathrm{t})} \mathrm{S}_{(\mathrm{t})}-\varepsilon \mathrm{E}_{(\mathrm{t})} \\
\frac{\mathrm{dI}(\mathrm{t})}{\mathrm{dt}}=\varepsilon \mathrm{E}_{(\mathrm{t})}-\sigma \mathrm{I}_{(\mathrm{t})} \\
\frac{\mathrm{dR}_{(\mathrm{t})}}{\mathrm{dt}}=\sigma \mathrm{I}_{(\mathrm{t})} \\
\mathrm{S}_{0}, \mathrm{E}_{0}, \mathrm{I}_{0}, \mathrm{R}_{0,} \lambda, \varepsilon, \sigma>0 \\
\mathrm{~S}_{(\mathrm{t})}+\mathrm{E}_{(\mathrm{t})}+\mathrm{I}_{(\mathrm{t})}+\mathrm{R}_{(\mathrm{t})}=1
\end{array}\right.
$$

Where: $\mathrm{S}(\mathrm{t})$ is the rate of healthy people in the crowd at time t. Since Ebola infectious ability is very strong, so every healthy is also the susceptible people. $\mathrm{E}(\mathrm{t})$ is the rate of people during the incubation period at time t. In the continuous model, this paper must consider the period from incubation period to being confirmed infected. $\mathrm{I}(\mathrm{t})$ is the rate of people having been infected in the crowd at time $t$. $R(t)$ is the rate of people removing the infecting system at time $t$, which includes the people having recovered and dead.

I know that if the number of healthy and the infected people get larger, people can be easier to be infected. That to say, during the unit time, the number of susceptible people decreases more quickly. This paper use const $\lambda$ to represent the proportionality coefficient. When the susceptible crowd comes into the incubation period, the rate of people in the incubation period will increase and also decrease because it can transformed into infected period with a certain proportion. The more people are in incubation period, the more quickly its amount can decrease. Thus, $\mathrm{E}(\mathrm{t})$ increase rate is inversely proportional to $\mathrm{E}(\mathrm{t})$. The paper uses the const $\varepsilon$ to represent the coefficient. In the same way, there will be people in incubation period with $\varepsilon \mathrm{E}(\mathrm{t})$ rate coming into the infected period within unit time. Besides, the $\mathrm{R}(\mathrm{t})$ increase rate is inversely proportional to $\mathrm{R}(\mathrm{t})$. This paper use const $\sigma$ to represent the coefficient. Finally, as for the fourth equation above, this paper know that people removing from the SEIR system are the people dead and having recovered.

These people come from the infected crowd. Therefore, its increase rate is proportional to I(t). And then, this paper comes to cope with the value determination of those three parameters.

$$
\varepsilon=\frac{1}{\mathrm{n}} \sum_{\mathrm{i}=1}^{\mathrm{n}} \frac{\Delta \mathrm{E}_{\mathrm{i}} / \Delta \mathrm{t}}{\mathrm{E}_{\mathrm{i}}} \quad \text { (1) } \quad \sigma=\frac{1}{\mathrm{n}} \sum_{\mathrm{i}=1}^{\mathrm{n}} \frac{\Delta \mathrm{R}_{\mathrm{i}} / \Delta \mathrm{t}}{\mathrm{I}_{\mathrm{i}}}
$$

$\lambda$ : Through numerous trials to get the most optimal solution.

\section{2Solving process of the model}

From the data we've collected, this paper uses the equation 1,2 and gets the parameter $\varepsilon, \sigma$ solution for every country and the totality.

Table 2: The solution of parameter $\varepsilon$ and $\sigma$

\begin{tabular}{|c|cccc|}
\hline & Totality & Guinea & Liberia & Sierra Leone \\
\hline$\varepsilon$ & 0.0603 & 0.2619 & 0.0097 & 0.1993 \\
\hline$\sigma$ & 0.0233 & 0.0597 & 0.0142 & 0.0241 \\
\hline
\end{tabular}

Although the Ebola has also broken out in other many countries except Guinea, Liberia and Sierra Leone. But such as in Nigeria, there are 22 confirmed cases since 10th September 2014. But then, no new case has been found. Besides, a country can be considered to be free of Ebola virus transmission when 42 days has elapsed since the last patient gets the isolation. Therefore, this paper only considers these three countries which are in bad condition in this model and their sum as the totality. Since the data is adequate from the time of August 2014, $\varepsilon$ and $\sigma$ can be considered to be relatively accurate.

Next, this paper put the value of $\varepsilon$ and $\sigma$ into this SEIR model. And as for $\lambda$ this paper tries the value from 0 to 1 ; getting the estimated value $\left.\left.\mathrm{I}_{\mathrm{i}}\right)^{2}+\left(\begin{array}{c}\widehat{\mathrm{R}} \\ \mathrm{l}\end{array}-\mathrm{R}_{\mathrm{i}}\right)^{2}\right\}$ and getting $\lambda$, solution as follows:

$$
\begin{gathered}
\widehat{I} \\
(\mathrm{t}) \\
\text { ws: }
\end{gathered}
$$


Table 3: The solution of parameter $\lambda$

\begin{tabular}{|l|cccc|}
\hline & Totality & Guinea & Liberia & Sierra Leon \\
\hline$\lambda$ & 0.1500 & 0.1680 & 0.1440 & 0.1460 \\
\hline
\end{tabular}

Thus, the three parameters in this SEIR model have been determined. Through this model, this paper can predict the trends of $\mathrm{S}(\mathrm{t}), \mathrm{E}(\mathrm{t}), \mathrm{I}(\mathrm{t}), \mathrm{R}(\mathrm{t})$ and the value in the future at any time $\mathrm{t}$ (unit time is a week). The trends curve can be observed in the following figure.

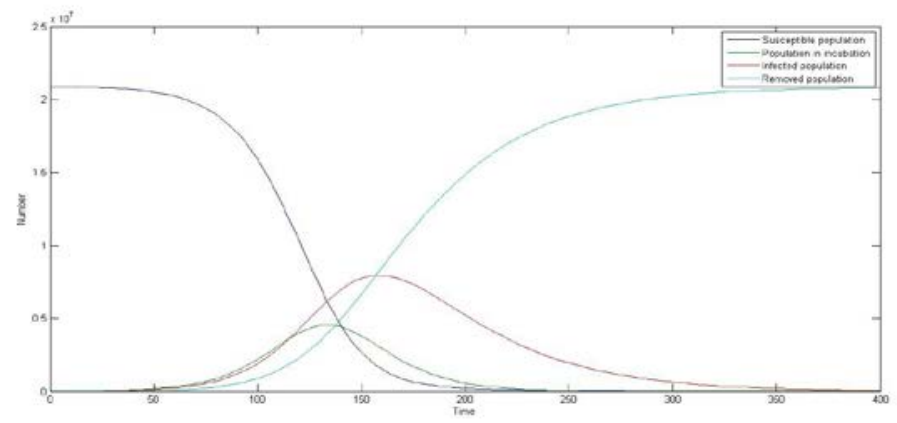

Figure 1: The prediction figure of Ebola total epidemic situation

As this paper can see from the trend figure, this paper can find without human intervention by drugs or vaccines, the rate of infected people will increase continuously and became stable in the end. This paper can find the best point of putting into drug and control the epidemic situation. This can be very useful for determining the right time to distribute drug and vaccine since they are all limited especially the drug. In total, this SEIR can predict the epidemic situation change trend very properly. However, the value prediction can bring relatively large error. Then, this paper employs the Grey Prediction Model to solve it.

\section{3Value estimation with Grey Prediction}

In the real life, this paper can find that for some cases, this paper can obtain all information this paper want and some cases contrarily no information at all. And for most cases, this paper can only collect partial information. The first kind is called the white system, the second black system and the third grey system. Grey Prediction is the process of analysis, model building, model solving and prediction for the grey system applying the grey model GM $(1, \mathrm{~N})$. Here, the information of Ebola outbreak till now has been collected. Thus, value prediction can be relatively easy.

This paper take the I(t) for example. This paper assume the original data $\mathrm{I}(\mathrm{t})$ in different week makes up the sample sequence $\mathrm{x}^{(0)}=\left\{\mathrm{x}_{1}{ }^{(0)}, \mathrm{x}_{2}{ }^{(0)} \ldots \ldots \mathrm{x}_{\mathrm{k}}{ }^{(0)}\right\}$. Through a procession of the data in the sequence, new sequence can be produced. Having more data, this paper can dig out and find the regularity among the real data. In general, this paper can choose the way of cumulative generation, regressive generation and so on. This paper choose the common method-cumulative generation.

$$
\mathrm{x}_{\mathrm{k}}{ }^{(1)}=\sum_{\mathrm{m}=1}^{\mathrm{k}} \mathrm{x}_{\mathrm{m}}{ }^{(0)}(\mathrm{k}=1,2, \ldots, \mathrm{n})
$$

Thus, get the new sequence: $\mathrm{x}_{\mathrm{k}}{ }^{(1)}=\left\{\mathrm{x}_{1}{ }^{(1)}, \mathrm{x}_{2}{ }^{(1)} \ldots \ldots \mathrm{x}_{\mathrm{k}}{ }^{(1)}\right\}$

In a similar way, have the non-mean generation: $\mathrm{z}_{\mathrm{k}}{ }^{(0)}=\alpha \mathrm{x}_{\mathrm{k}+1}{ }^{(0)}+(1-\alpha) \mathrm{x}_{\mathrm{k}-1}{ }^{(0)}, 0<\alpha<1$

Build the first-order linear differential white model on the sequence $\mathrm{x}_{\mathrm{k}}{ }^{(1)}$

$$
\frac{\mathrm{dx}}{\mathrm{dt}}^{(1)}+\alpha \mathrm{x}^{(1)}=\mathrm{u}
$$

Employing least square method, this paper can solve parameter a, u and the analytical expression:

$$
\widehat{x_{k+1}^{(1)}}=\left(x_{1}{ }^{(0)}-\frac{a}{u}\right) e^{-a k}+\frac{a}{u}, k=1,2, \cdots n
$$

From this, this paper can get the estimated value for the original data. When satisfying the following equation:

$$
\min \left\{\sum_{\mathrm{i}=1}^{\mathrm{n}} \frac{\left|\widehat{\mathrm{x}}_{1}^{(0)}-\mathrm{x}_{\mathrm{i}}^{(0)}\right|}{\mathrm{x}_{\mathrm{i}}^{(0)}}\right\}
$$


Then, get the optimal $\alpha$ for equation 4.3, and this prediction is relatively most accurate. Through using the model for all data, this paper have obtained $\mathrm{S}(\mathrm{t}), \mathrm{E}(\mathrm{t}), \mathrm{I}(\mathrm{t}), \mathrm{R}(\mathrm{t})$ data in two weeks in those three country and totality.

Table 4: $\mathrm{S}(\mathrm{t})$ value in next two weeks

\begin{tabular}{|l|cccl|}
\hline & Totality & Guinea & Liberia & Sierra Leone \\
\hline Week 1 & $20,859,000$ & $10,495,000$ & $4,281,500$ & $6,082,700$ \\
\hline Week 2 & $20,858,000$ & $10,494,000$ & $4,281,200$ & $6,082,300$ \\
\hline
\end{tabular}

Table 5: $\mathrm{E}(\mathrm{t})$ value in next two weeks

\begin{tabular}{|l|lclc|}
\hline & Totality & Guinea & Liberia & Sierra Leone \\
\hline Week 1 & 8,968 & 376 & 5,776 & 2,823 \\
\hline Week 2 & 9,280 & 392 & 5,933 & 2,967 \\
\hline
\end{tabular}

Table 6: I(t)value in next two weeks

\begin{tabular}{|l|cccc|}
\hline & Totality & Guinea & Liberia & Sierra Leone \\
\hline Week 1 & 23,027 & 3,033 & 8,930 & 11,076 \\
\hline Week 2 & 23,514 & 3,090 & 9,089 & 11,356 \\
\hline
\end{tabular}

Table 7: R(t)value in next two weeks

\begin{tabular}{|l|lrrc|}
\hline & Totality & Guinea & Liberia & Sierra Leone \\
\hline Week 1 & 9,244 & 2,005 & 3,809 & 3,408 \\
\hline Week 2 & 9,471 & 2, 056 & 3, 878 & 3, 518 \\
\hline
\end{tabular}

In this solving process, this paper calculates the relative error of each case. All of them are $10^{-5}$ order of magnitude. Thus, the accuracy of this prediction is round. With the original data and the predicted value, this paper gets the four sets of data trends graph. According to the principle of this model and the optimistic medication measures later, the prediction can only be limited for the near term. This paper combines this with this SEIR prediction. Right time for distribution can be determined as well as the allocation amount for each region. This paper can see the relatively accurate trends in the following figures. Since S(t) owns much higher magnitude than other three sets of data, this paper put them in separate graphs.

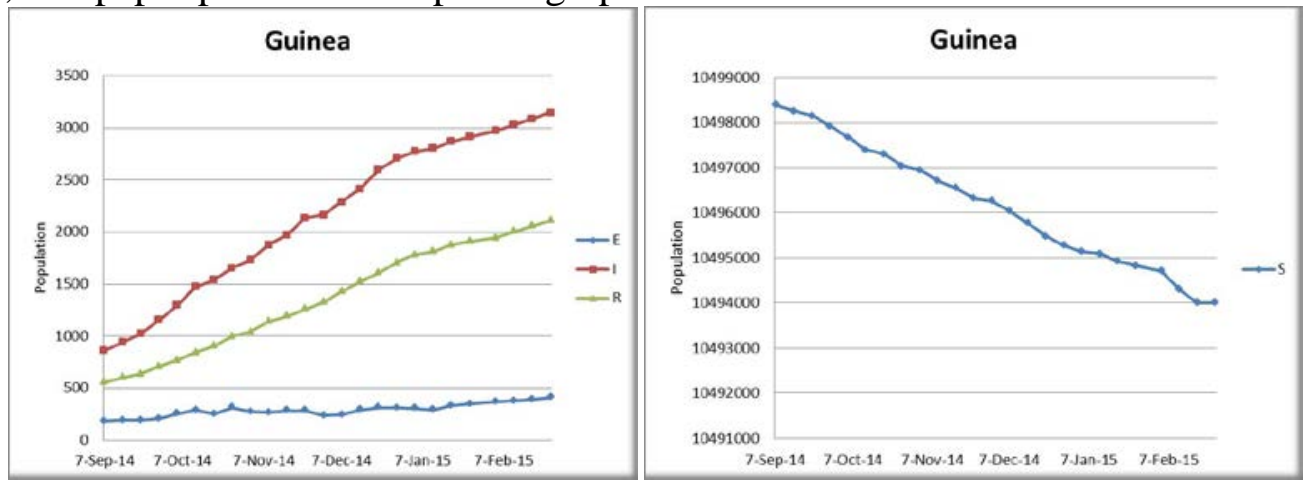

Fig. S, E, I, R trend in Guinea

From the figure, this paper can see the data of E, I, R show the continuous increase. Prediction is in accordance with the trend which original data indicates. The population of infected population increases most quickly. Thus, vaccines must be put into use as soon as possible. However, from the Fig. 1, the best control time point is before the future 50 weeks. This paper can find that population in incubation doesn't show much fluctuation. Actually, in this SEIR model, Et is a continuous parameter. And the incubation period of Ebola in human body is generally 2 21 days. Thus, the trend is reasonable. In the same way, this paper can get the trend in Liberia, Sierra Leone and the totality. 


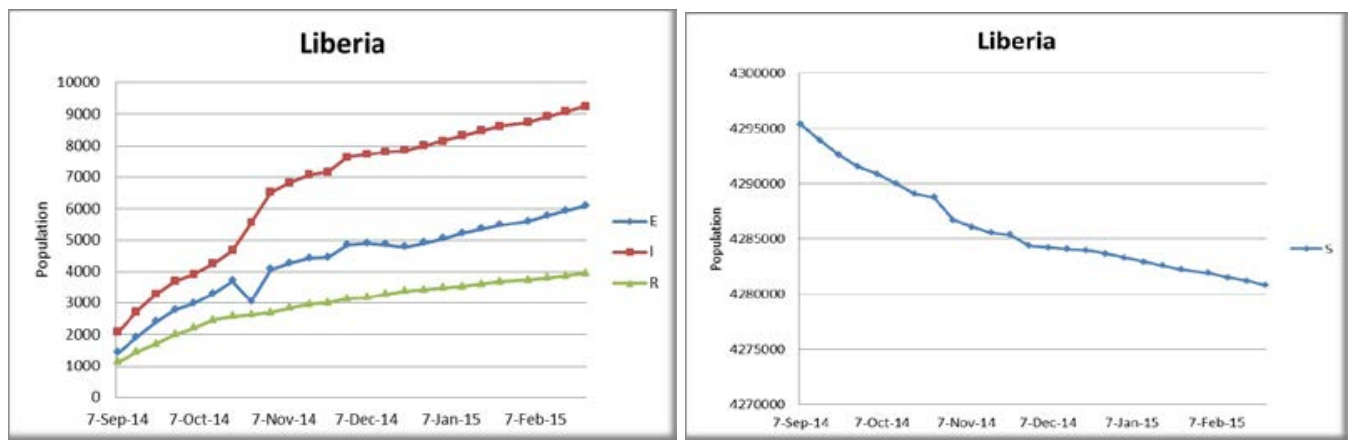

Fig. S, E, I, R trend in Liberia

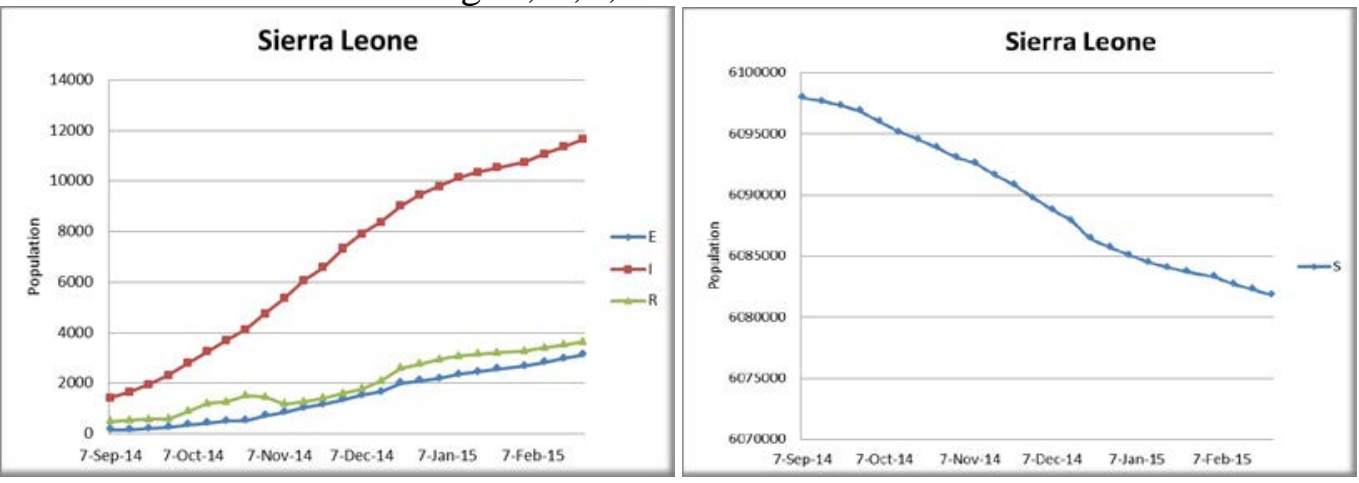

Fig. S, E, I, R trend in Sierra Leone

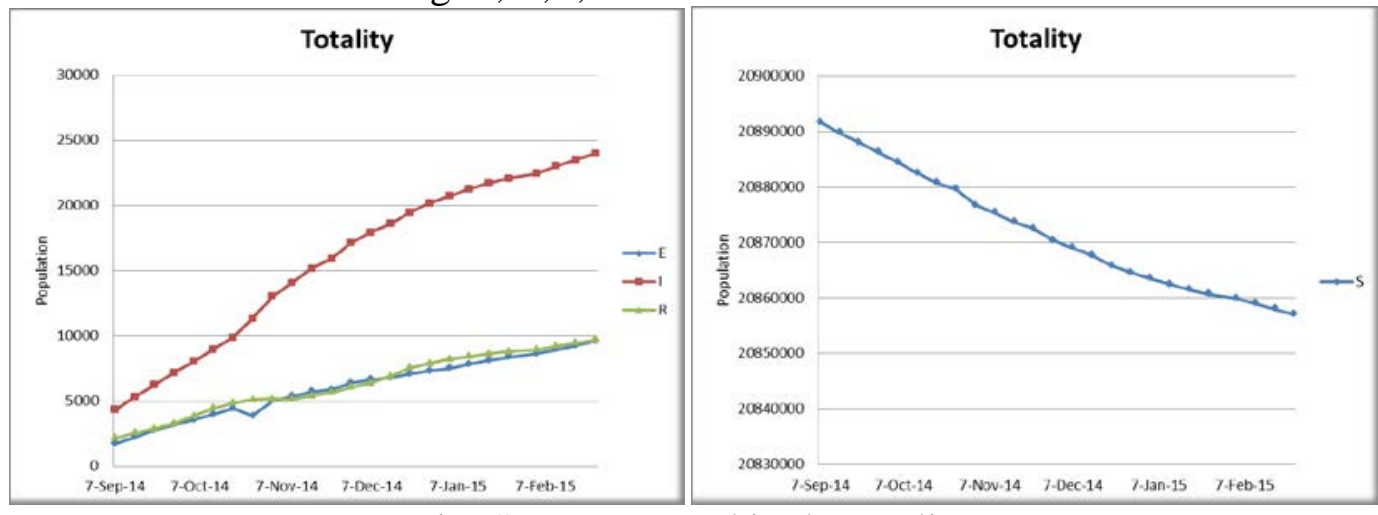

Fig. S, E, I, R trend in the totality

\section{Conclusions}

From the models discussed above, this paper can see that the current situation of Ebola is still unpleasant. This model can be concluded as: During normal days, this transport network work just as Fig. a, Fig. b, Fig. c show, every state has a nearby transfer station which provides the daily drugs and vaccine and is distributed according to the populations and the current situation.

\section{The Test of Model: Sensitiveness analysis}

This paper separately vary the three parameters $\lambda, \varepsilon, \sigma$, and then analyze the tendency of the SEIR model. This paper found that this model is very insensitive to these parameters as this paper only observes the tendency of this model, regardless of the time. However, this paper builds this model to predict the tendency of the Ebola transmission, so as to provide a strategy for an optimal time to control.

\section{Acknowledgment}

This work was financially supported by Anqi Li. 


\section{References}

[1] Xvdong Cao, Youwen Li, Hongbin zhang. Mathematical Modeling and theMethod [M]. Beijing: Higher Education Press, 2014:41 44, 275 281.

[2] http://apps. who. int/gho/data/view. ebola-sitrep. ebola-summary-20141112?lang=en

[3] Zhizong Chen, Jianxin You. Muti-objuective Decision Model of Facility Location in Emergency Rescue[J]. Management Sciences in China, 2006, 19[4]:1 5.

[4] http://en. wikipedia. org/wiki/Guinea

[5] http://en. wikipedia. org/wiki/Liberia 